\title{
Scattering by stochastic boundaries: hybrid low- and high-order quantification algorithms \\ M. Ganesh ${ }^{1} \quad$ S. C. Hawkins ${ }^{2}$
}

(Received 18 February 2015; revised 11 January 2016)

\begin{abstract}
We present an efficient framework for simulating the average wave scattering properties of two dimensional randomly shaped particles with statistical properties similar to model aerosols particles that are important in atmospheric science applications. Our framework is based on an efficient high order discretisation of the spatial dimensions and parallel implementations for the large number of stochastic dimensions. We demonstrate our framework by simulating the mean (and higher order moments) of the far field of the model particles. We use tens of thousands of Monte Carlo, quasi-Monte Carlo and sparse grid generalised polynomial chaos realisations of the random particle model.
\end{abstract}

http://journal . austms.org.au/ojs/index.php/ANZIAMJ/article/view/9313 gives this article, (c) Austral. Mathematical Soc. 2016. Published February 3, 2016, as part of the Proceedings of the 17th Biennial Computational Techniques and Applications Conference. ISSN 1446-8735. (Print two pages per sheet of paper.) Copies of this article must not be made otherwise available on the internet; instead link directly to this URL for this article. 


\section{Contents}

1 Introduction

C313

2 Model stochastic particles and properties

C316

3 Scattering model and numerical method

C320

4 Numerical experiments

C323

5 Conclusions

C331

References

C333

A Proof of approximation

C335

\section{Introduction}

Simulation of the average scattering properties of randomly shaped particles is important in many applications. One example concerns the light scattering properties of ice crystals and atmospheric aerosol particles, which are important in climate science because of their impact on radiative transfer in the atmosphere $[1,8,10,16]$.

We focus on two dimensional simulations of the average far field of particles with random shapes and properties similar to those used in atmospheric science $[10,16]$. Tackling two dimensional models is an important stepping stone towards developing efficient hybrid determinisitic-stochastic algorithms for solving the full three dimensional model for feldspar aerosol particles $[1,16]$, which will be the subject of our future work.

The main quantity of interest in scattering models is the stochastic complex valued far field $\mathfrak{u}^{\infty}$, defined as a function of the observed direction and the stochastic variable. For example, in climate science, key data such as the 
scattering cross section of particles and the extinction coefficients are derived from the far field [15, p. 12].

Following the work of Nousiainen and McFarquhar [10] and Veihelmann et al. [16] on ice crystals and dust particles, Section 2 describes a stochastic model for randomly shaped particles $\mathrm{D}(\boldsymbol{\omega})$, where $\boldsymbol{\omega}$ is an element of the set of outcomes $\Omega$ in a probability space $(\Omega, \mathcal{F}, \mathrm{P})$, and $\mathcal{F}$ is a $\sigma$-algebra of $\Omega$, with $\mathrm{P}$ the associated probability measure. We denote by $\mathfrak{u}^{\infty}(\cdot ; \boldsymbol{\omega})$ the far field induced by the interaction of an incident plane wave with the particle $\mathrm{D}(\boldsymbol{\omega})$.

Our main focus is to compute the expected value of the intensity of the far field

$$
\mathbb{E}\left[\left|u^{\infty}(\widehat{x} ; \cdot)\right|^{2}\right]=\int_{\Omega}\left|u^{\infty}(\widehat{x} ; \boldsymbol{\omega})\right|^{2} \mathrm{dP}(\boldsymbol{\omega}), \quad \widehat{x} \in \partial B,
$$

where $\partial \mathrm{B}$ is the unit circle comprising all observation direction vectors $\widehat{\boldsymbol{x}}$. The direction $\widehat{\mathbf{d}}$ in which the incident plane wave propagates is considered fixed. Section 3 describes a high-order numerical method for the stochastic wave propagation model. The numerical solution exactly satisfies the radiation condition.

Lamberg et al. [9], Nousiainen and McFarquhar [10] and Veihelmann et al. [16] investigated randomly shaped scattering particles with $\Omega=\mathbb{R}^{S}$ and Gaussian probability measure where $S$ is the stochastic model order and is typically a few tens in the two dimensional case (and a few hundreds in the three dimensional case). In this work we consider the interesting and practical case of randomly shaped particles with bounded $\Omega \subset \mathbb{R}^{S}$ and probability measure associated with the uniform distribution.

In both the Gaussian and uniform distribution cases, the high dimension of the integral in (1) requires efficient (finite sum) approximations with a trade-off between the accuracy of evaluating the integral and a practical number of cubature/realization points. For each cubature point, the full scattering model needs to be solved to compute the associated far field. 
In this work we approximate the integral (1) using state of the art Monte Carlo (MC), quasi-Monte Carlo (QMC) and sparse-grid generalised polynomial chaos (sg-gPC) techniques. Le Maître and Kino [13] and recent reviews [5, 12, and extensive references] provided details of these three stochastic approximation methods. All three approaches lead to tens of thousands of independent scattering realizations with fixed scatterers. We simulate each of these realizations using an efficient high-order Nyström scheme [3] with high-order quadrature to evaluate weakly-singular integrals.

The modified Nyström scheme is equivalent to a pseudo-spectral algorithm [6] and hence, unlike standard Nyström techniques, leads to high-order convergence. For each realization of the random model, the number of degrees of freedom for simulating the far field depends on the roughness of the scattering particle. The Nyström scheme leads to relatively smaller linear systems than low-order boundary/finite element methods (that do not exploit the special structure of the stochastic particles considered in this article). Thus the algorithms employed here include a hybrid of low/high-order stochastic sampling and high-order spatial discretization. The independence of the large number of spatial scattering simulations allows further acceleration by taking advantage of parallel computing.

Our work is closely related to several interesting articles. In particular, Veihelmann et al. [16] used the low order discrete dipole method for scattering simulations with $100 \mathrm{MC}$ samples to approximate the scattering properties of model feldspar particles, and Nousiainen and McFarquhar [10] used a similar random particle model for atmospheric ice crystals and the ray optics approximation for particles that are large compared with the incident wavelength.

Using our efficient computational framework, Section 4 presents results obtained using tens of thousands of realizations to compare the industrial standard MC, QMC, and sg-gPC high-dimensional stochastic integral approximations for application to scattering by stochastic boundaries. We are not aware of any previous numerical results that combine such large scale 
stochastic sampling with efficient high-order stochastic boundary scattering simulations for this important class of model particles. Previous approaches involve low-order scattering simulations (based on perturbation methods, the method of moments, and the finite element method) applied in combination with MC, QMC, and high order integration methods (including gPC) for scatterers whose randomness consists of random deformations of a fixed shape $[2,11,14,17]$.

\section{Model stochastic particles and properties}

It is standard in scattering theory to parametrise the boundary of two dimensional particles using polar coordinates [3]. We parametrise the stochastic boundary $\partial \mathrm{D}(\boldsymbol{\omega})$ of the model particle $\mathrm{D}(\boldsymbol{\omega})$ using $\mathbf{q}_{\boldsymbol{\omega}}:[0,2 \pi) \rightarrow \partial \mathrm{D}(\boldsymbol{\omega})$, where

$$
\mathbf{q}_{\boldsymbol{\omega}}(\theta)=\mathbf{r}_{\boldsymbol{\omega}}(\theta)(\cos \theta, \sin \theta), \quad \theta \in[0,2 \pi) .
$$

We follow the approach of Nousiainen and McFarquhar [10] and Veihelmann et al. [16] to imbue the stochastic radius function with certain statistical properties. These properties were chosen so that the resulting stochastic shapes model various kinds of particles analysed in atmospheric science using instruments such as a cloud particle imager $[1,10,16]$.

The stochastic radius function is

$$
r_{\boldsymbol{\omega}}(\theta)=\frac{1}{\sqrt{1+\sigma^{2}}} \exp \left[s_{\boldsymbol{\omega}}(\theta)\right], \quad \theta \in[0,2 \pi),
$$

where $\sigma^{2}$ is a physical parameter of the model, $\mathrm{N}$ is a truncation parameter, and the log-radius function is represented by a stochastic truncated Fourier (or Karhunen-Loéve) expansion:

$$
s_{\boldsymbol{\omega}}(\theta)=\sum_{k=2}^{N} a_{k} \cos k \theta+\sum_{k=2}^{N} b_{k} \sin k \theta .
$$


The coefficients in (4) are independent (uniform or Gaussian) random variables with zero mean, and the variance of $a_{k}$ and $b_{k}$ is $\delta_{k}^{2}$ with

$$
\delta_{k}=\alpha k^{-m}, \quad \text { for } k=2, \ldots, N .
$$

Here $m$ is a parameter that controls the roughness of the particle boundary, and $\alpha$ is a normalisation parameter chosen so that

$$
\sum_{k=2}^{N} \delta_{k}^{2}=\delta^{2}, \quad \delta^{2}=\log \left(1+\sigma^{2}\right) .
$$

Thus the dimension of the associated probability space $\Omega$ is $S=2 \mathrm{~N}-2$ and we write $\boldsymbol{\omega}=\left(a_{2}, \ldots, a_{N}, b_{2}, \ldots, b_{N}\right)$.

The random particles in the model are specified by the two parameters $\mathrm{m}$ and $\sigma$ (and the truncation parameter $\mathrm{N}$ ), and by the distribution of the random variables associated with the coefficients. For the simulations we choose $m=5 / 2$ and $\sigma=1 / 5$ so that in the Gaussian case our random particles model feldspar dust [10, 16]. Figures 1 and 2 visualise samples of the uniform random particle model with $\mathrm{N}=5$ and $\mathrm{N}=10$. The particles are blobby for small $\mathrm{N}$ and become rougher with increasing $\mathrm{N}$.

Next we outline some statistical properties of the log-radius and radius. We are not aware of any results giving these statistical properties when the coefficients $a_{k}$ and $b_{k}$ in (4) are uniform random variables. Equivalent properties for the Gaussian case are given by Lamberg et al. [9].

First we consider the covariance of the log-radius, which is independent of the distribution type. Using the independence of the zero mean random variables 
Figure 1: Samples of the uniform random particle model with $N=5$ and hence there are $S=8$ random variables. The particles are blobby for small $\mathrm{N}$ and become rougher with increasing $\mathrm{N}$.
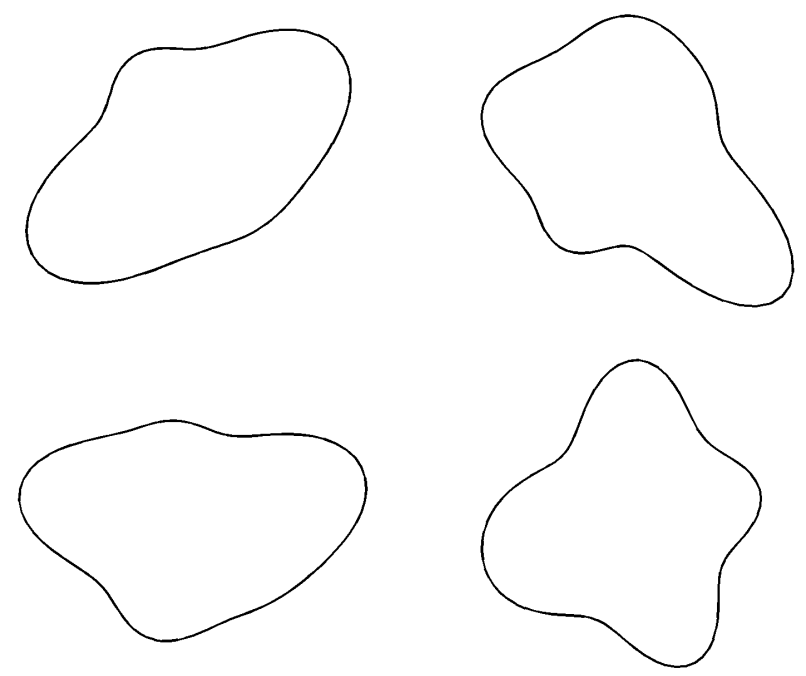

in (4),

$$
\begin{aligned}
\operatorname{Cov}\left[s_{\boldsymbol{\omega}}\left(\theta_{1}\right), s_{\boldsymbol{\omega}}\left(\theta_{2}\right)\right]= & \mathbb{E}\left[s_{\boldsymbol{\omega}}\left(\theta_{1}\right) s_{\boldsymbol{\omega}}\left(\theta_{2}\right)\right] \\
= & \mathbb{E}\left[\left(\sum_{j=2}^{N} a_{j} \cos j \theta_{1}+\sum_{j=2}^{N} b_{j} \sin j \theta_{1}\right)\right. \\
& \left.\times\left(\sum_{k=2}^{N} a_{k} \cos k \theta_{2}+\sum_{k=2}^{N} b_{k} \sin k \theta_{2}\right)\right] \\
= & \sum_{k=2}^{N} \mathbb{E}\left[a_{k}^{2}\right] \cos k \theta_{1} \cos k \theta_{2}+\sum_{k=2}^{N} \mathbb{E}\left[b_{k}^{2}\right] \sin k \theta_{1} \sin k \theta_{2} .
\end{aligned}
$$


Figure 2: Samples of the uniform random particle model with $N=10$ and hence there are $S=18$ random variables. The particles are blobby for small $\mathrm{N}$ and become rougher with increasing $\mathrm{N}$.
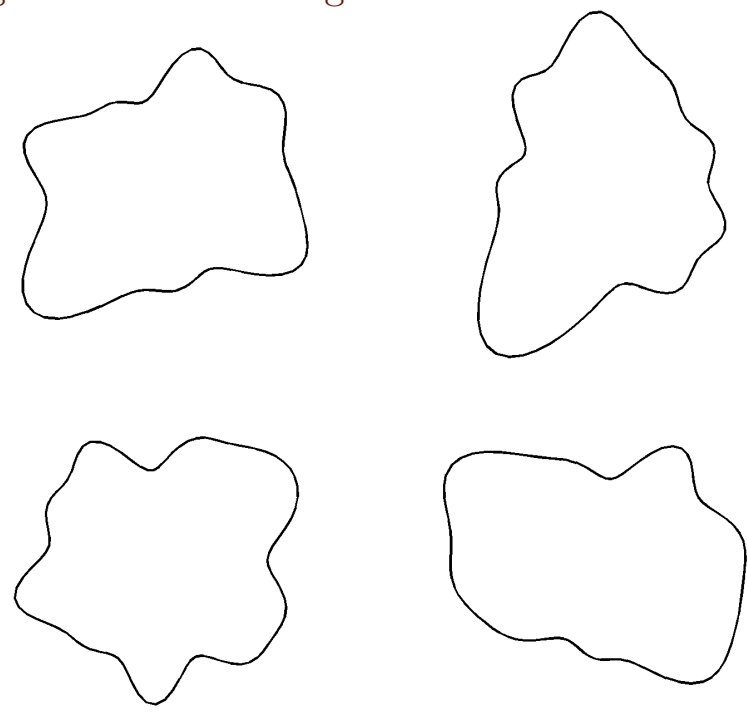

Consequently, using the double angle formula for cosine,

$$
\operatorname{Cov}\left[s_{\boldsymbol{\omega}}\left(\theta_{1}\right), s_{\boldsymbol{\omega}}\left(\theta_{2}\right)\right]=\sum_{k=2}^{N} \delta_{k}^{2} \cos k\left(\theta_{1}-\theta_{2}\right) .
$$

Thus the variance of the log-radius is $\delta^{2}$.

Uniform random particle case: When the coefficients $a_{k}$ and $b_{k}$ in (4) are uniform random variables, the covariance has no simple representation. However, Appendix A shows that the mean of the radius is approximately one, and derives the approximation

$$
\operatorname{Cov}\left[\mathrm{r}_{\boldsymbol{\omega}}\left(\theta_{1}\right), \mathrm{r}_{\boldsymbol{\omega}}\left(\theta_{2}\right)\right] \approx \exp \left\{\operatorname{Cov}\left[\mathbf{s}_{\boldsymbol{\omega}}\left(\theta_{1}\right), \boldsymbol{s}_{\boldsymbol{\omega}}\left(\theta_{2}\right)\right]\right\}-1
$$


These approximations are valid for small $\delta_{2}, \ldots, \delta_{N}$ and it follows that for sufficiently small $\sigma^{2}$ the variance of the radius is approximately $\exp \left(\delta^{2}\right)-1=$ $\sigma^{2}$.

Gaussian random particle case: When the coefficients $a_{k}$ and $b_{k}$ in (4) are Gaussian random variables, the statistical properties of the radius are known [9]. In particular, the radius has unit mean and covariance

$$
\operatorname{Cov}\left[r_{\boldsymbol{\omega}}\left(\theta_{1}\right), \boldsymbol{r}_{\boldsymbol{\omega}}\left(\theta_{2}\right)\right]=\exp \left\{\operatorname{Cov}\left[s_{\boldsymbol{\omega}}\left(\theta_{1}\right), \boldsymbol{s}_{\boldsymbol{\omega}}\left(\theta_{2}\right)\right]\right\}-1 .
$$

Thus the variance of the radius is $\exp \left(\delta^{2}\right)-1=\sigma^{2}$ and the parameter $\sigma^{2}$ has physical significance. Lamberg et al. $[9, \S 2]$ give an equivalent covariance (9), after taking into account the normalized radius in (3).

\section{$3 \quad$ Scattering model and numerical method}

We assume that the model stochastic particle $D(\boldsymbol{\omega})$ is illuminated by a time-harmonic incident plane wave with wavelength $\lambda$. The incident plane wave is represented by its complex valued spatial component

$$
u^{\text {inc }}(\boldsymbol{x})=e^{i \kappa x \cdot \widehat{d}},
$$

where the fixed unit vector $\widehat{\mathbf{d}}$ represents the direction of propagation of the incident wave and $k=2 \pi / \lambda$ is the wavenumber. The interaction of the incident wave with the particle $\mathrm{D}(\boldsymbol{\omega})$ induces a complex valued stochastic scattered field $\mathfrak{u}(\cdot ; \boldsymbol{\omega})$ satisfying the Helmholtz equation

$$
\Delta u(x ; \boldsymbol{\omega})+\kappa^{2} u(x ; \boldsymbol{\omega})=0, \quad x \in \mathbb{R}^{2} \backslash \bar{D}(\boldsymbol{\omega}),
$$

and the Sommerfeld radiation condition [3, eq. (3.85)]

$$
\lim _{|x| \rightarrow \infty} \sqrt{|x|}\left(\frac{\partial u}{\partial x}(x ; \boldsymbol{w})-i \kappa u(x ; \boldsymbol{w})\right)=0
$$


uniformly with respect to the direction $\boldsymbol{x} /|\boldsymbol{x}| \in \partial \mathrm{B}$. It is well known that the Maxwell model for light scattering in the two dimensional case, with appropriate polarization, reduces to solving the Helmholtz equation [3].

To simplify the discussion, we consider only the stochastic sound-soft particle $\mathrm{D}(\boldsymbol{\omega})$ and hence on the boundary $\partial \mathrm{D}(\boldsymbol{\omega})$ of $\mathrm{D}(\boldsymbol{\omega})$ we apply the vanishing total field boundary condition

$$
u(x ; \boldsymbol{\omega})+u^{\text {inc }}(\boldsymbol{x})=0, \quad x \in \partial D(\boldsymbol{\omega}) .
$$

The techniques presented in this work are readily modified for other boundary conditions (for example, impedance and transmission boundary conditions).

A consequence of the radiation condition (11) is that [3, eq. (3.86)]

$$
u(x ; \boldsymbol{\omega})=\frac{e^{i \kappa|x|}}{\sqrt{|x|}}\left[u^{\infty}(\widehat{x} ; \boldsymbol{\omega})+O\left(\frac{1}{|x|}\right)\right],
$$

for $\widehat{\boldsymbol{x}}=\boldsymbol{x} /|\boldsymbol{x}|$ as $|\boldsymbol{x}| \rightarrow \infty$. The complex valued stochastic far field $u^{\infty}$ defined on the unit circle $\partial \mathrm{B}$ and its first stochastic moment given by (1) are typically the quantities of interest in applications.

Our approach for approximating the expected value integral (1) has two parts. The first part is a cubature rule for the high dimensional integral (1) that requires evaluation of the far field for each cubature point $\boldsymbol{\omega}_{1}, \ldots, \boldsymbol{\omega}_{M} \in$ $\Omega$. The second part is to approximate the far field of the deterministic particle $\mathrm{D}\left(\boldsymbol{\omega}_{\mathrm{j}}\right)$ for each of the cubature points $\boldsymbol{j}=1, \ldots, M$. Since a far field computation is required for each of the $M$ cubature points, it is crucial that this is performed efficiently.

For a fixed particle $\mathrm{D}\left(\boldsymbol{\omega}_{j}\right)$ we use a surface integral representation of the scattered field

$$
u(\boldsymbol{x} ; \boldsymbol{\omega})=\int_{\partial \mathrm{D}\left(\boldsymbol{w}_{j}\right)}\left[\frac{\partial \mathrm{G}}{\partial \mathbf{n}(\mathbf{y})}(\boldsymbol{x}, \mathbf{y})-\mathfrak{i}_{\kappa} \mathrm{G}(\boldsymbol{x}, \mathbf{y})\right] \phi(\mathbf{y}) \mathrm{ds}(\mathbf{y})
$$

where

$$
\mathrm{G}(\boldsymbol{x}, \boldsymbol{y})=\frac{i}{4} \mathrm{H}_{0}^{(1)}(\kappa|\boldsymbol{x}-\mathbf{y}|)
$$


is the free space Green's function for the two dimensional Helmholtz equation and $\mathrm{H}_{0}^{(1)}$ is the zero order Hankel function of the first kind. The unknown boundary potential $\phi$ in (14) satisfies the second kind integral equation

$$
\phi(x)+2 \int_{\partial \mathrm{D}\left(\boldsymbol{\omega}_{j}\right)}\left[\frac{\partial \mathrm{G}}{\partial \mathfrak{n}(\mathbf{y})}(\boldsymbol{x}, \mathbf{y})-i_{\kappa} \mathrm{G}(\boldsymbol{x}, \mathbf{y})\right] \phi(\mathbf{y}) \mathrm{ds}(\mathbf{y})=-2 \mathrm{u}^{\mathrm{inc}}(\boldsymbol{x}),
$$

for $\boldsymbol{x} \in \partial \mathrm{D}\left(\boldsymbol{\omega}_{j}\right)$. The scattered field (14) exactly satisfies the Helmholtz equation (10) and the radiation condition (11) [3].

We numerically solve the second kind integral equation (15) using the efficient high-order Nyström scheme $[3, \S 3.5]$ using $2 \mathfrak{n}$ quadrature points on $\partial \mathrm{D}\left(\boldsymbol{\omega}_{\mathrm{j}}\right)$ and a high-order quadrature rule for the weakly-singular integral in (15). The resulting approximate potential is $\phi_{n}$. For smooth scatterers, such as those in our random particle model, this scheme exhibits high-order convergence of $\phi_{\mathrm{n}}$ to $\phi$ (in the maximum norm) with order constant that depends mainly on the roughness of the stochastic boundary. Hence the high-order method requires only to resolve the roughness in the stochastic boundary, leading to relatively small linear systems that can be solved quickly using a direct solver. This is the key to the efficiency of our hybrid numerical method for the model problem described, with large stochastic dimension and unbounded exterior spatial region.

Using $\phi_{\mathrm{n}}$ and the ansatz (14) we obtain a boundary integral representation for the approximate far field [3, eq. (3.87)]

$$
u_{n}^{\infty}(\widehat{\boldsymbol{x}} ; \boldsymbol{\omega})=\frac{e^{i \pi / 4}}{\sqrt{8 \pi \kappa}} \int_{\partial \mathrm{D}\left(\boldsymbol{\omega}_{j}\right)}\left[\frac{\partial e^{-i \kappa \widehat{x} \cdot \mathbf{y}}}{\partial \mathfrak{n}(\mathbf{y})}-i \kappa e^{-i \kappa \widehat{x} \cdot \mathbf{y}}\right] \phi_{n}(\mathbf{y}) \mathrm{ds}(\mathbf{y})
$$

To approximate (1) for fixed $\widehat{\boldsymbol{x}} \in \mathrm{\partial B}$ we use the cubature rule

$$
\mathbb{E}_{M}\left[\left|u_{n}^{\infty}(\widehat{x} ; \boldsymbol{\omega})\right|^{2}\right]=\sum_{j=1}^{M} v_{j}\left|u_{n}^{\infty}\left(\widehat{x} ; \boldsymbol{\omega}_{j}\right)\right|^{2},
$$

with cubature points $\boldsymbol{\omega}_{1}, \ldots, \boldsymbol{\omega}_{M}$ and weights $v_{1}, \ldots, \boldsymbol{v}_{M}$. The numerical far field simulations for the cubature points in (17) are independent and so in our implementation we efficiently perform these in parallel. 
In our numerical experiments we use three cubature sampling rules.

MC: Low-order rule with equal weights $v_{j}=1 / M$ for $j=1, \ldots, M$ and cubature points taken from a random sample of $\boldsymbol{\omega}$.

QMC: High-order rules based on the Sobol points using equal weights $v_{j}=$ $1 / M$ for $j=1, \ldots, M$ and low discrepancy quadrature points [4] with interlace factor $\mathrm{d}=1, \ldots, 4$.

sg-gPC: High-order rule using a Smölyak sparse-grid [7] with Gauss-Hermite or Gauss-Legendre quadrature in the Gaussian and uniform cases, respectively.

Remark 1. The generalised polynomial chaos approach computes a high order orthogonal polynomial approximation to $\left|\boldsymbol{u}_{n}^{\infty}(\widehat{\boldsymbol{x}} ; \boldsymbol{w})\right|^{2}$. We focus on the expected value, which is obtained from the zero order coefficient and is mathematically equivalent to the cubature rule (17). Le Maitre and Kino [13] provided full details.

Remark 2. The (full-grid) gPC is practical if the number of random variables $S$ is fewer than five. The sparse-grid gPC provides a framework for the case $S>5$ at the expense of reduced accuracy. For larger $S$, say $S>15$, the MC and QMC realizations are appropriate because the rate of convergence of the $\mathrm{MC} / \mathrm{QMC}$ is expected to be independent of (or mildly dependent on) S. Proving the MC, QMC, and sg-gPC theoretical rates of convergence and quantification of the associated order constants for the wave propagation model is an open problem. The proofs will depend crucially on the regularity of the quantity of interest in appropriate norms. These proofs will be part of our future work.

\section{Numerical experiments}

In this section we demonstrate the hybrid numerical approach for the stochastic wave scattering model (10)-(12) with stochastic boundaries obtained using parameters $\mathrm{N}=5,10$ (corresponding to the stochastic dimension $S=8,18$ ) 
by simulating the average far field induced by the model random particles (see Figures 1 and 2) with diameters one and four times the incident wavelength. It is useful to investigate the behavior of the hybrid algorithms as the incident frequency increases, especially to understand the low-order MC and high-order QMC approximations of the stochastic integrals for large stochastic dimensional wave propagation models.

For each fixed value of the stochastic parameter $\boldsymbol{\omega}$, the Nyström scheme parameter $\boldsymbol{n}$ required to attain a given accuracy in the far field $\boldsymbol{u}_{n}^{\infty}$ depends (in a complicated way) on the incident wavenumber $k$ and on the shape of the scatterer. In the case of our stochastic particle model, the scatterer shape is random, and care must be taken to ensure that the parameter $n$ is sufficiently large to attain the desired accuracy for all samples of the random particle model.

To investigate appropriate values of $n$, for each of the problems considered in this section we measured the error for $1000 \mathrm{MC}$ samples of the random particle. The true value of the far field is not known, so we use the standard approach of approximating the error using a reference solution computed with more degrees of freedom. Since the method is spectrally accurate, a moderate increase in $\boldsymbol{n}$ is sufficient to obtain a comparison solution. In particular, for a fixed realisation $\boldsymbol{\omega}$ of the random model and a test value of $n$, our relative error estimate for $\boldsymbol{u}_{n}^{\infty}(\cdot ; \boldsymbol{\omega})$ is

$$
\rho_{n}(\boldsymbol{\omega})=\frac{\left\|u_{n}^{\infty}(\cdot ; \boldsymbol{\omega})-u_{n+5}^{\infty}(\cdot ; \boldsymbol{\omega})\right\|_{\infty}}{\left\|u_{n+5}^{\infty}(\cdot ; \boldsymbol{\omega})\right\|_{\infty}} .
$$

We approximate the maximum norm on $\partial B$ in $\rho_{n}$ by taking the maximum error at 1000 observation angles. The histograms in Figures 3 and 4 show the normalised frequency against the logarithm (base 10) of the error for $1000 \mathrm{MC}$ samples of the uniform random particle model with $S=8$ and $S=18$. These histograms establish that (with very high probability) we obtain at least six digits accuracy in our simulation of the associated exterior scattering model for each stochastic boundary realization. 
Figure 3: Histograms showing the normalised frequency against the logarithm (base 10) of the error in the computed far field $\boldsymbol{u}_{n}^{\infty}(\cdot ; \boldsymbol{\omega})$ for 1000 samples of $\boldsymbol{\omega}$ for the random particle model with $S=8$ for (top) average diameter $\lambda$ and $n=35$; (bottom) average diameter $4 \lambda$ and $n=80$.
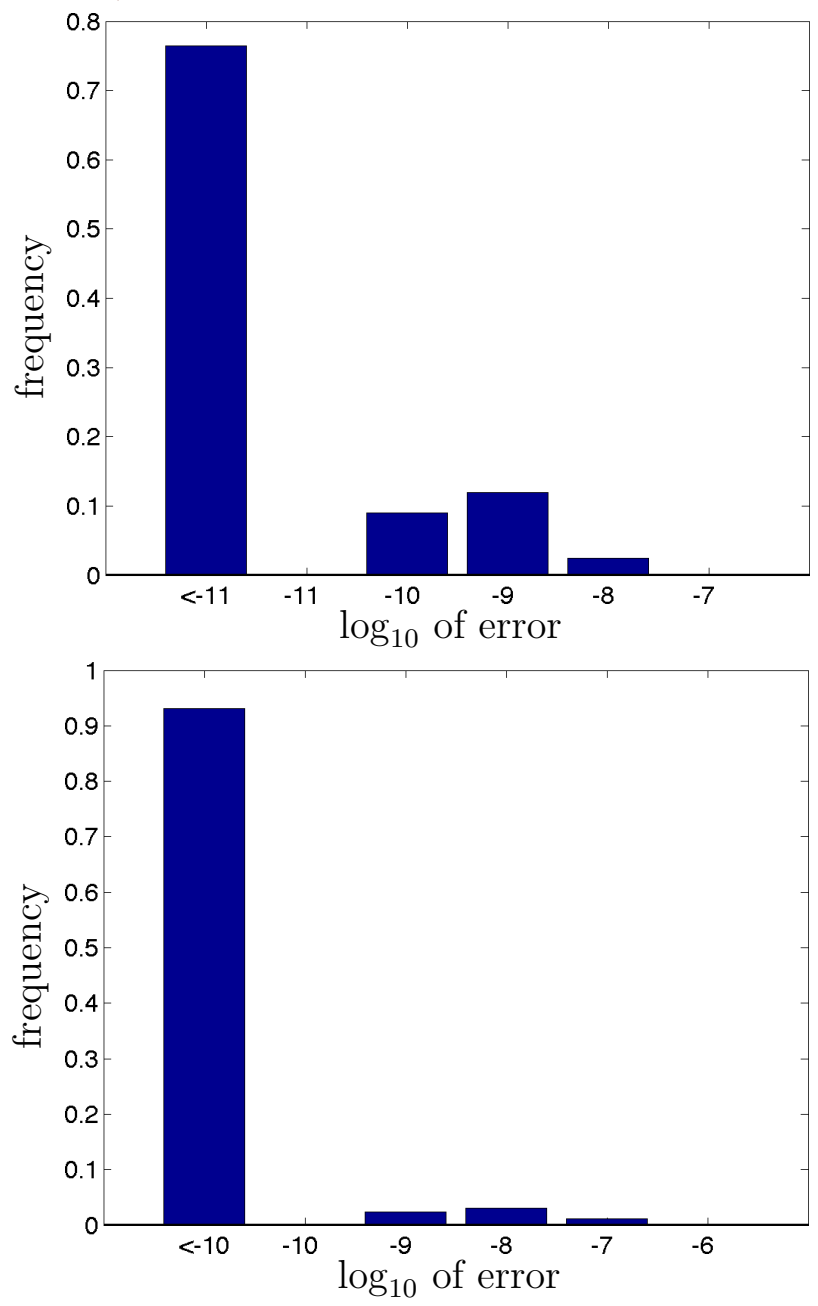
Figure 4: Histograms showing the normalised frequency against the logarithm (base 10) of the error in the computed far field $\mathfrak{u}_{n}^{\infty}(\cdot ; \boldsymbol{\omega})$ for 1000 samples of $\boldsymbol{\omega}$ for the random particle model with $S=18$ for (top) average diameter $\lambda$ and $n=35$; (bottom) average diameter $4 \lambda$ and $n=80$.
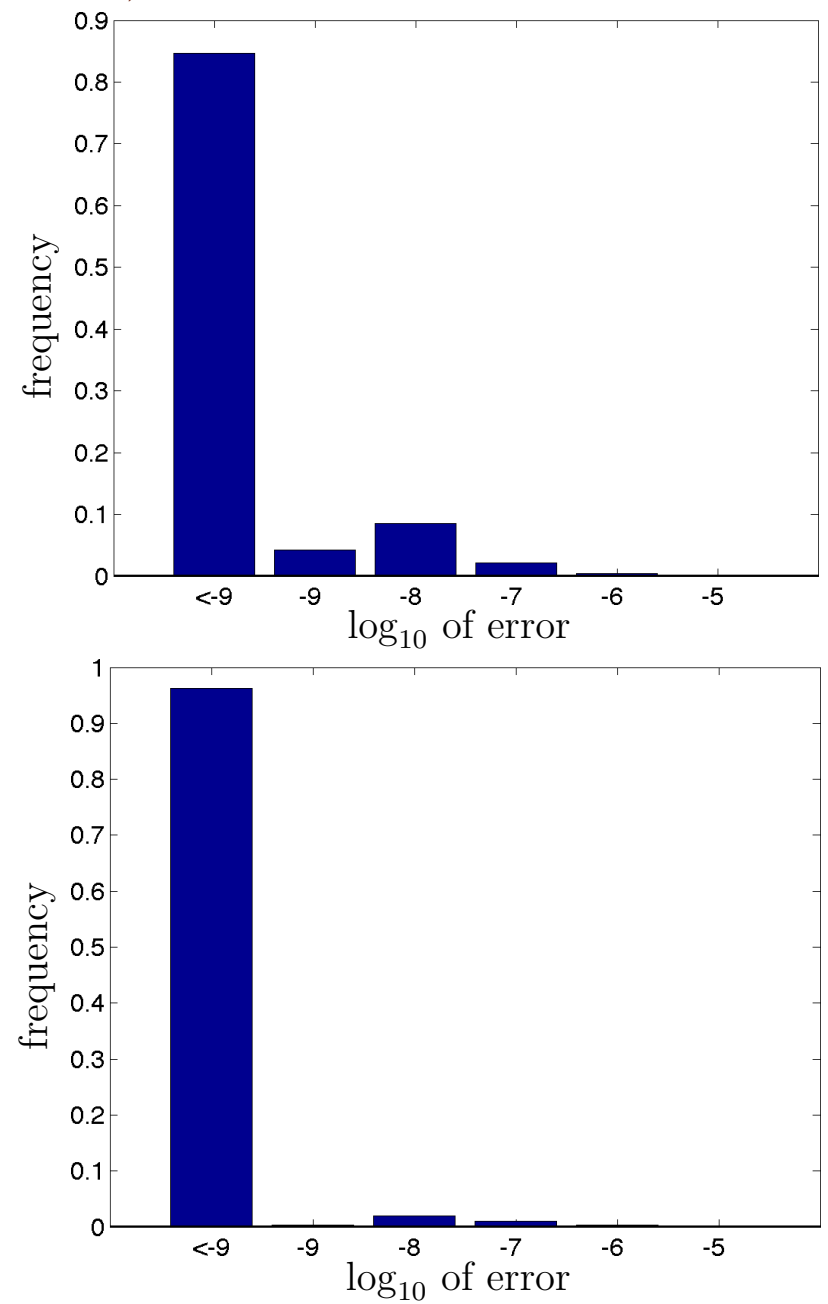
Table 1: Parallel CPU time required for $M=65536 \mathrm{MC}$ simulations for the uniform random particle model with $S=18$ random variables and Nyström parameter $\mathrm{n}$ on two $2.5 \mathrm{GHz}$ ten-core Intel Xeon processors for particles with mean diameter one and four times the incident wavelength $\lambda$.

\begin{tabular}{rrr} 
diameter & $\mathrm{n}$ & CPU time $(\mathrm{min})$ \\
\hline$\lambda$ & 55 & 33.7 \\
$4 \lambda$ & 110 & 128.3
\end{tabular}

The principal part of the CPU time required by our algorithm is that required to perform the $M$ independent scattering simulations and is proportional to $M$. Hence the $\mathrm{CPU}$ time is essentially independent of which cubature rule is used. Table 1 demonstrates the efficiency of our parallel algorithm by tabulating the parallel $\mathrm{CPU}$ time required to perform $\mathrm{M}=2^{16}=65536 \mathrm{MC}$ simulations for the uniform random particle model with $S=18$.

For the uniform particle model with $\mathrm{N}=5$ the stochastic dimension $\mathrm{S}=8$ is sufficiently small that we are able to use the sg-gPC scheme. Table 2 gives the relative error in the expected value of the far field computed using the sg-gPC scheme for the uniform random particle model for particles of mean diameter equal to the incident wavelength. Figure 5 plots the relative error in the expected value of the far field computed using the MC and QMC schemes for the uniform random particle model with $S=8$ for particles of mean diameter equal to one incident wavelength. For this particle we compare all three cubature models and Figure 6 again plots the relative error in the expected value of the far field computed using the MC and QMC (with interlace parameter $\mathrm{d}=4$ ) and sg-gPC schemes. The error plotted is measured in the infinity norm, approximated at 1000 equally spaced observation angles. Because the exact value of the mean is not known, for the error calculations we took as our reference solution the mean far field computed using the QMC scheme with $M=2^{16}=65536$ points and interlace parameter $d=4$.

Figures 7 and 8 plot the relative error in the expected value of the far field, computed using the MC and QMC schemes for the uniform random particle 
Table 2: Relative error in the computed expected value of the far field for the sparse grid generalised polynomial chaos scheme with $M$ points for the uniform random particle model with $S=8$. The mean diameter of the particles equals the incident wavelength. The Nyström parameter $n=35$.

\begin{tabular}{rrr} 
gPC degree & $M$ & rel. error \\
\hline 2 & 145 & $2.8 \times 10^{-3}$ \\
4 & 3905 & $5.1 \times 10^{-4}$ \\
6 & 51713 & $1.8 \times 10^{-4}$
\end{tabular}

Figure 5: Relative error in the computed expected value of the far field plotted against $\log _{2} M$ for MC and QMC schemes with $M$ points for the uniform random particle model with $S=8$ random variables. The mean diameter of the particles equals one incident wavelength. The Nyström parameter $\mathrm{n}=35$.

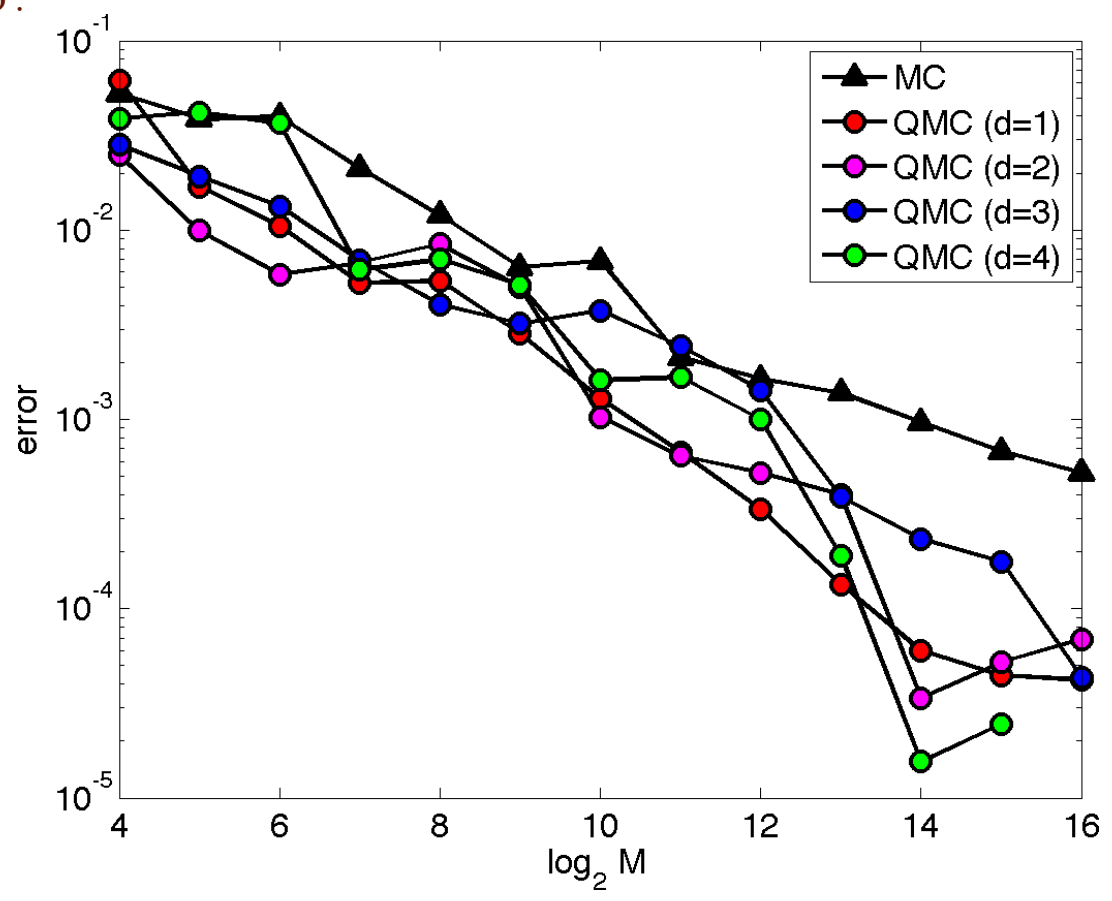


Figure 6: Relative error in the computed expected value of the far field plotted against $\log _{2} M$ for MC, QMC and sparse grid gPC schemes with $M$ points for the uniform random particle model with $S=8$ random variables. The mean diameter of the particles equals one incident wavelength. The Nyström parameter $n=35$.

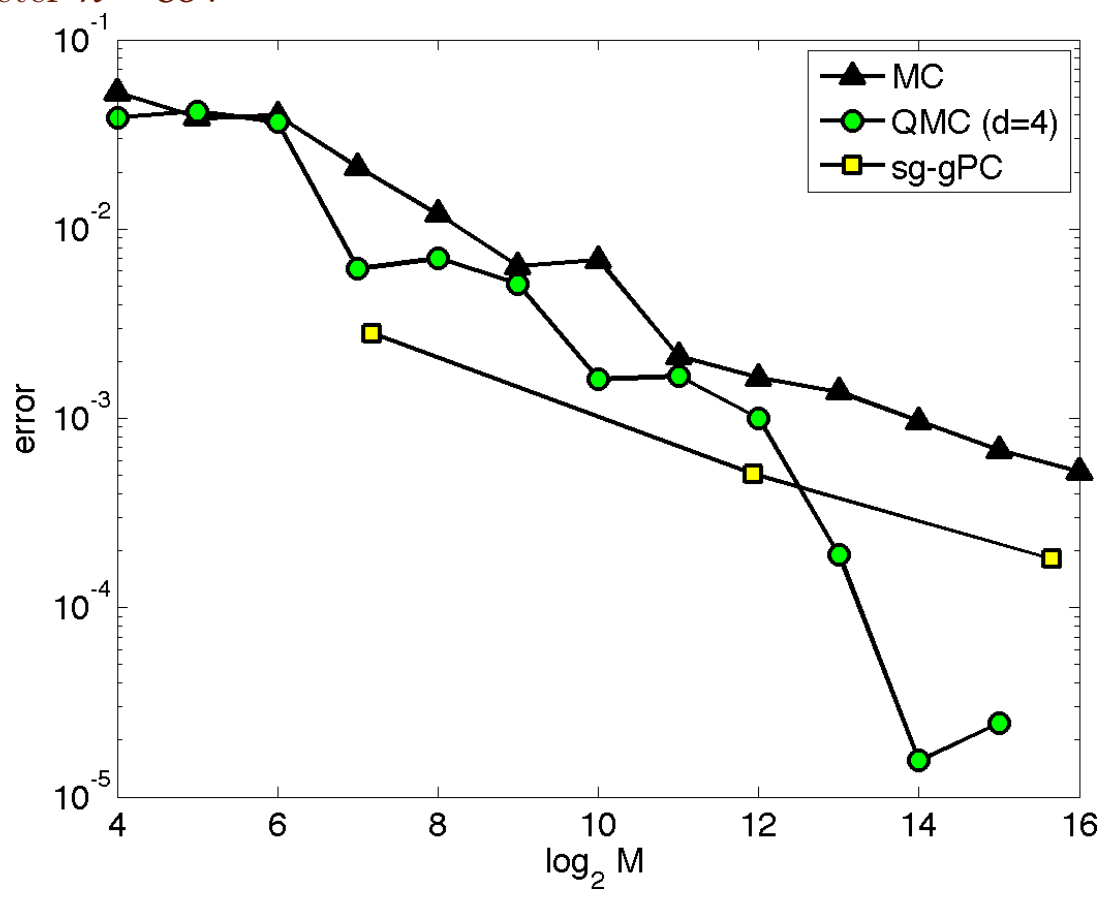

model with $S=18$ and for particles of mean diameter one and four times the incident wavelength. Again, we took as our reference solution the mean far field computed using the QMC scheme with $M=2^{16}=65536$ points and interlace parameter $\mathrm{d}=4$.

Finally, Figure 9 visualises the mean and standard deviation of $\left|\mathfrak{u}_{n}^{\infty}(\cdot ; \boldsymbol{\omega})\right|^{2}$ by plotting the cross section of the mean far field for the uniform random particle model with $S=18$. The mean and standard deviation are obtained using the QMC method with $M=2^{16}=65536$ points and interlace parameter $d=4$. 
Figure 7: Relative error in the computed expected value of the far field plotted against $\log _{2} M$ for $M C$ and QMC schemes with $M$ points for the uniform random particle model with $S=18$ random variables. The mean diameter of the particles equals one incident wavelength. The Nyström parameter $\mathrm{n}=55$.

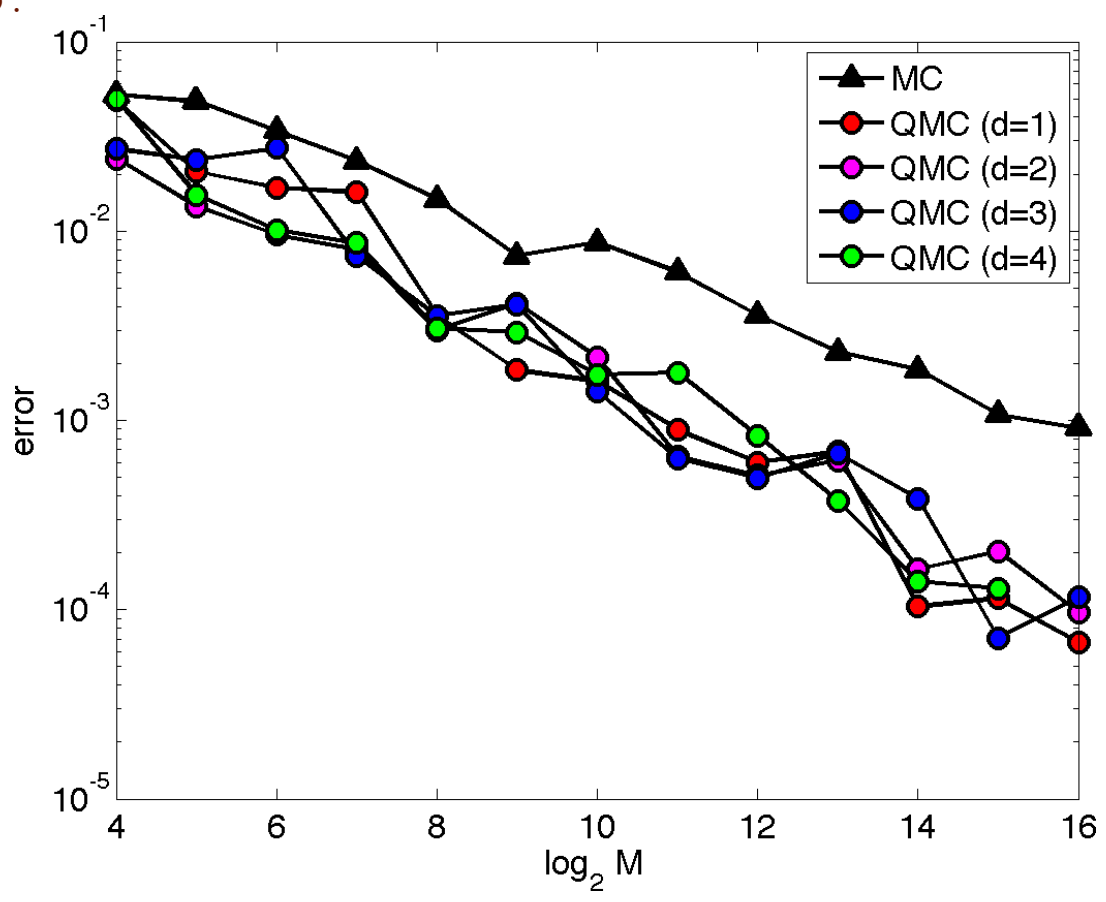

The cross section, in decibels, is obtained from the far field by applying

$$
\rho^{\mathrm{dB}}(u)=10 \log _{10} 2 \pi|u|^{2} .
$$


Figure 8: Relative error in the computed expected value of the far field plotted against $\log _{2} M$ for $M C$ and QMC schemes with $M$ points for the uniform random particle model with $S=18$ random variables. The mean diameter of the particles equals four incident wavelength. The Nyström parameter $\mathrm{n}=110$.

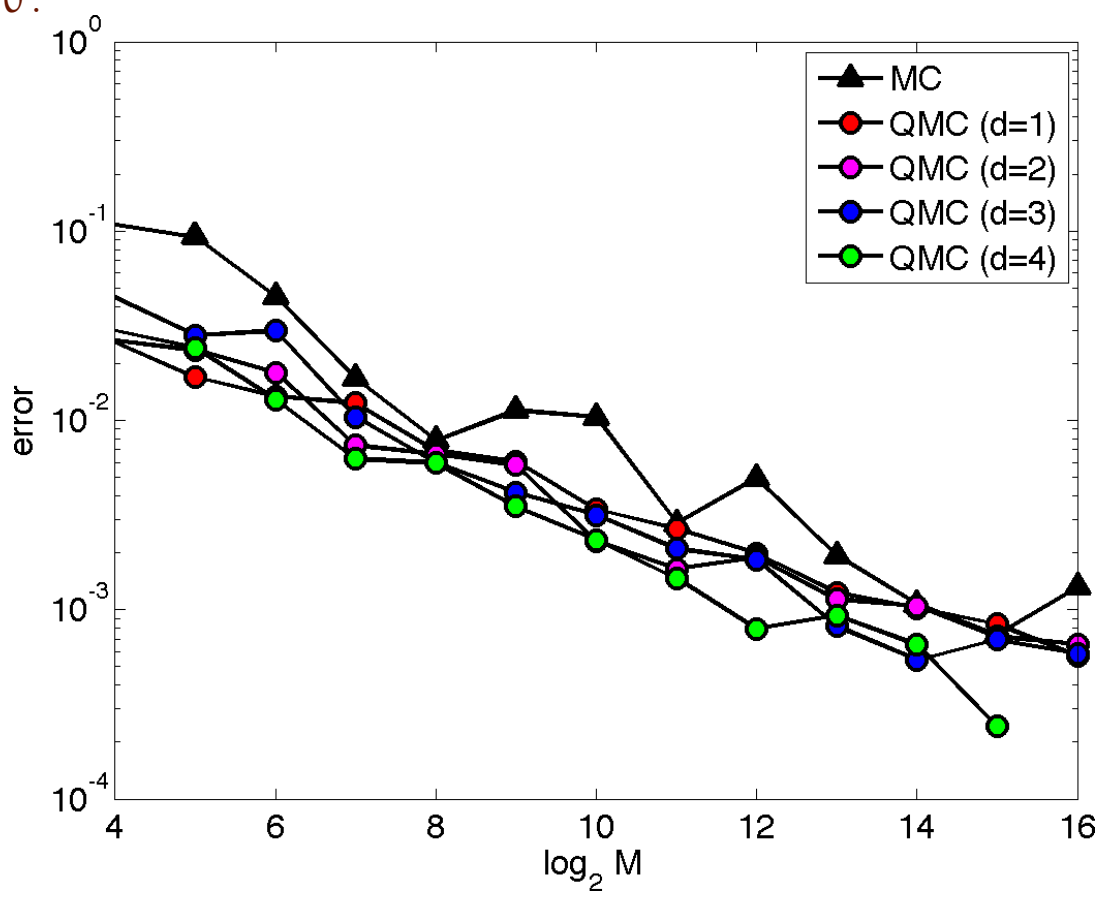

\section{Conclusions}

The development of advanced uncertainty quantification algorithms for scattering by randomly shaped particles is at an early stage. There are several unresolved issues, including theoretical results on the smoothness of the quantities of interest as functions of the random variables in the stochastic model; sharp frequency dependent convergence estimates for QMC schemes for high dimension problems; and computational issues related to the large stochastic 
Figure 9: Mean far field (in decibels) obtained using the QMC method with $M=65536$ simulations and interlace parameter $d=4$ for the uniform random particle model with $S=18$ random variables and incident wave coming from the direction $0^{\circ}$. The shaded region indicates the mean plus and minus one standard deviation. The mean diameter of the particles is (top) one incident wavelength; (bottom) four times the incident wavelength.

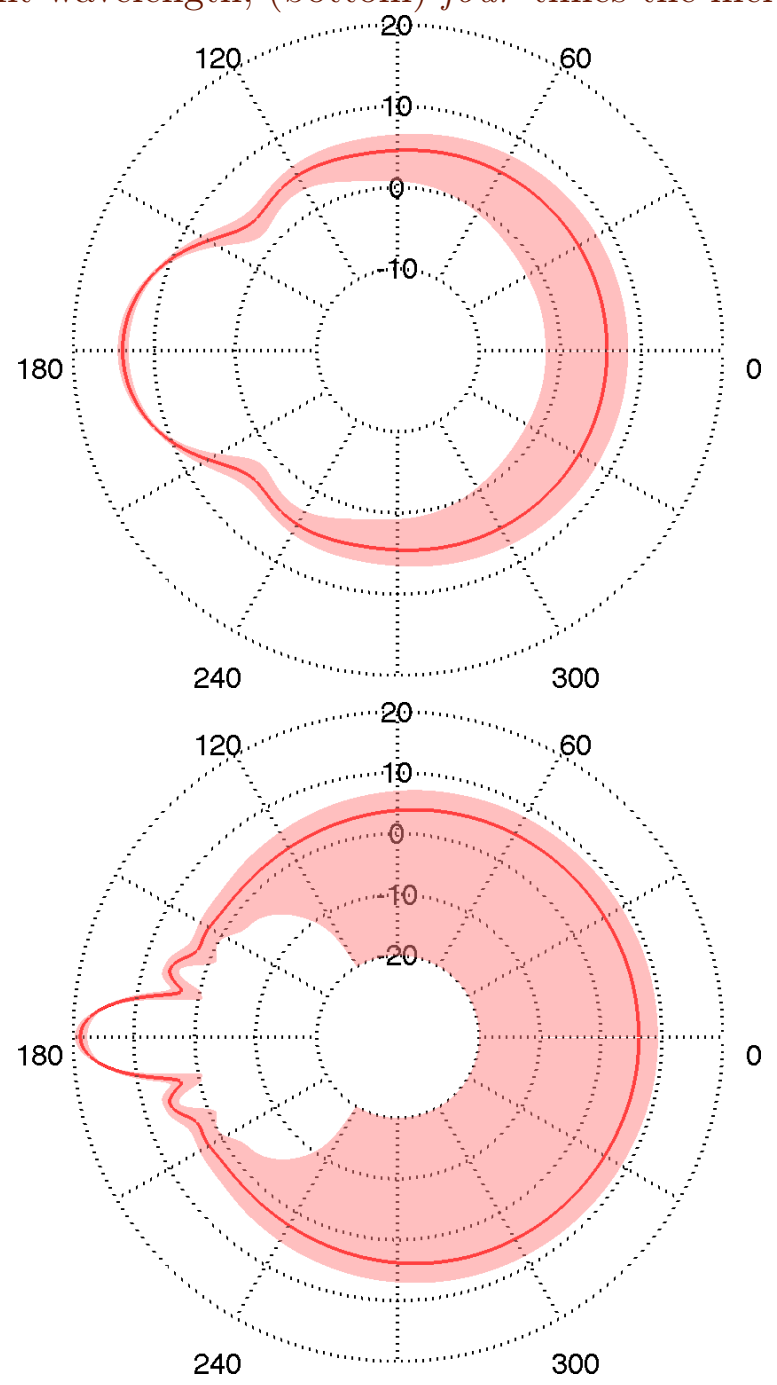


dimension of the problem. This work represents a substantial step forward for some of these issues. In particular, our efficient computational framework facilitates large scale simulations that are necessary for the investigation of smoothness and convergence. This is a key stepping stone towards simulating the full three dimensional model, which has important applications.

The numerical results in this work suggest that for low frequency problems the QMC method converges at a faster rate than the MC method, but that the convergence rate of the QMC method decreases as the frequency increases. Our experiments suggest that the standard deviation of the far field also increases as the frequency increases. It is likely that these two phenomena are connected. This illustrates the extreme challenge posed by uncertainty quantification in wave scattering applications for high incident frequencies.

Acknowledgment The research of the M. Ganesh was supported, in part, by grant DMS-1216889 from the National Science Foundation. Support of the Colorado Golden Energy Computing Organization is gratefully acknowledged.

\section{References}

[1] A. J. Baran. From the single-scattering properties of ice crystals to climate prediction: A way forward. Atmos. Res., 112:45-69, 2012. doi:10.1016/j.atmosres.2012.04.010 C313, C316

[2] C. Chauviere, J. Hesthaven, and L. Wilcox. Efficient computation of RCS from scatterers of uncertain shapes. IEEE T. Antenn. Propag., 55:1437-1448, 2007. doi:10.1109/TAP.2007.895629 C316

[3] D. Colton and R. Kress. Inverse Acoustic and Electromagnetic Scattering Theory. Springer, 2013. doi:10.1007/978-1-4614-4942-3 C315, C316, C320, C321, C322 
[4] J. Dick. Walsh spaces containing smooth functions and quasi-Monte Carlo rules of arbitrary high order. SIAM J. Numer. Anal., 46:1519-1553, 2008. doi:10.1137/060666639 C323

[5] J. Dick, F. Y. Kuo, and I. H. Sloan. High-dimensional integration: The quasi-Monte Carlo way. Acta Numer., 22:133-288, 2013. doi:10.1017/S0962492913000044 C315

[6] M. Ganesh and S. C. Hawkins. An efficient algorithm for simulating scattering by a large number of two dimensional particles. CTAC2010, ANZIAM J., 52:C139-C155, 2011. http://journal austms.org.au/ ojs/index.php/ANZIAMJ/article/view/3954 C315

[7] T. Gerstner and M. Griebel. Numerical integration using sparse grids. Numer. Algorithms, 18:209-232, 1998. doi:10.1023/A:1019129717644 C323

[8] P. V. Hobbs and M. P. McCormick. Aerosols and Climate. A. Deepak, 1988. http://catalogue.nla.gov.au/Record/514698 C313

[9] L. Lamberg, K. Muinonen, J. Ylönen, and K. Lumme. Spectral estimation of Gaussian random circles and spheres. J. Comput. Appl. Math., 136:109-121, 2001. doi:10.1016/S0377-0427(00)00578-1 C314, C317, C320

[10] T. Nousiainen and G. M. McFarquhar. Light scattering by quasi-spherical ice crystals. J. Atmos. Sci., 61:2229-2248, 2004. doi:10.1175/1520-0469(2004)061<2229:LSBQIC > 2.0.CO;2 C313, C314, C315, C316, C317

[11] O. Ozgun and M. Kuzuoglu. A coordinate transformation approach for efficient repeated solution of Helmholtz equation pertaining to obstacle scattering by shape deformations. Comput. Phys. Comm., 185:1616-1627, 2014. doi:10.1016/j.cpc.2014.03.002 C316 
[12] C. Schwab and C. J. Gittelson. Sparse tensor discretizations of high-dimensional parametric and stochastic PDEs. Acta Numer., 20:291-467, 2011. doi:10.1017/S0962492911000055 C315

[13] O. P. Le Maître and O. M. Kino. Spectral Methods for Uncertainty Quantification. Springer, 2010. doi:10.1007/978-90-481-3520-2 C315, C323

[14] P. Tsuji, D. Xiu, and L. Ying. Fast method for high-frequency acoustic scattering from random scatterers. Int. J. Uncertain. Quantif., 1:99-117, 2011. doi:10.1615/IntJUncertaintyQuantification.v1.i2 C316

[15] H. C. van de Hulst. Light Scattering by Small Particles. Dover, 1957. http://store.doverpublications.com/0486642283.html C314

[16] B. Veihelmann, T. Nousiainen, M. Kahnert, and W. J. van der Zande. Light scattering by small feldspar particles simulated using the Gaussian random sphere geometry. J. Quant. Spectrosc. Rad. Trans., 100:393-405, 2006. doi:10.1016/j.jqsrt.2005.11.053 C313, C314, C315, C316, C317

[17] D. Xiu and J. Shen. An efficient spectral method for acoustic scattering from rough surfaces. Commun, Comput. Phys., 2:54-72, 2007. http://www.global-sci.com/freedownload/v2_54.pdf C316

\section{A Proof of approximation}

In this appendix we derive the approximation (8) in Section 2 for the covariance of the radius

$$
\operatorname{Cov}\left[r_{\boldsymbol{\omega}}\left(\theta_{1}\right), r_{\boldsymbol{\omega}}\left(\theta_{2}\right)\right]=\mathbb{E}\left[\mathrm{r}_{\boldsymbol{\omega}}\left(\theta_{1}\right) \mathrm{r}_{\boldsymbol{\omega}}\left(\theta_{2}\right)\right]-\mathbb{E}\left[\mathrm{r}_{\boldsymbol{\omega}}\left(\theta_{1}\right)\right] \mathbb{E}\left[\mathrm{r}_{\boldsymbol{\omega}}\left(\theta_{2}\right)\right]
$$

The approximation is based on the variance of log-uniform random variables. In particular, for uniform random variables $X \sim \mathcal{U}(-\sqrt{3} \sigma, \sqrt{3} \sigma)$ with zero 
mean and variance $\sigma^{2}$, it is straightforward to derive the relation

$$
\mathbb{E}\left[e^{X}\right]=\frac{e^{\sqrt{3} \sigma}-e^{-\sqrt{3} \sigma}}{2 \sqrt{3} \sigma}=\frac{\sinh \sqrt{3} \sigma}{2 \sqrt{3} \sigma} .
$$

This is a special case of the moment generating function of $X$. Taylor's expansion about the origin gives

$$
\frac{\sinh \sqrt{3} \sigma}{2 \sqrt{3} \sigma}=e^{\sigma^{2} / 2}+\mathcal{O}\left(\sigma^{4}\right) .
$$

We begin by approximating the first term on the right hand side of (18). Using (3) we write the radius $\boldsymbol{r}_{\boldsymbol{\omega}}$ in terms of the log-radius $\mathbf{s}_{\boldsymbol{\omega}}$,

$$
\begin{aligned}
\mathbb{E}\left[r_{\boldsymbol{\omega}}\left(\theta_{1}\right) r_{\boldsymbol{\omega}}\left(\theta_{2}\right)\right] & =\frac{1}{1+\sigma^{2}} \mathbb{E}\left[\exp \left(\mathrm{s}_{\boldsymbol{\omega}}\left(\theta_{1}\right)\right) \exp \left(\mathrm{s}_{\boldsymbol{\omega}}\left(\theta_{2}\right)\right)\right] \\
& =\frac{1}{1+\sigma^{2}} \mathbb{E}\left[\exp \left(\mathrm{s}_{\boldsymbol{\omega}}\left(\theta_{1}\right)+\mathrm{s}_{\boldsymbol{\omega}}\left(\theta_{2}\right)\right)\right]
\end{aligned}
$$

Using the Fourier expansion of the log-radius (4), and collecting like terms we obtain

$$
s_{\boldsymbol{\omega}}\left(\theta_{1}\right)+s_{\boldsymbol{\omega}}\left(\theta_{2}\right)=\sum_{k=2}^{N} a_{k}\left(\cos k \theta_{1}+\cos k \theta_{2}\right)+\sum_{k=2}^{N} b_{k}\left(\sin k \theta_{1}+\sin k \theta_{2}\right) .
$$

The random variables $a_{2}, \ldots, a_{N}$ and $b_{2}, \ldots, b_{N}$ are independent of each other and hence the expected value in (21) decouples into factors,

$$
\begin{aligned}
\mathbb{E}\left[\exp \left(s_{\boldsymbol{\omega}}\left(\theta_{1}\right)+s_{\boldsymbol{\omega}}\left(\theta_{2}\right)\right)\right]= & \left(\prod_{k=2}^{N} \mathbb{E}\left[\exp \left(\mathrm{a}_{k}\left(\cos k \theta_{1}+\cos k \theta_{2}\right)\right)\right]\right) \\
& \times\left(\prod_{k=2}^{N} \mathbb{E}\left[\exp \left(b_{k}\left(\sin k \theta_{1}+\sin k \theta_{2}\right)\right)\right]\right) .
\end{aligned}
$$


Since $a_{k}\left(\cos k \theta_{1}+\cos k \theta_{2}\right)$ is a random variable with variance $\delta_{k}^{2}\left(\cos k \theta_{1}+\right.$ $\left.\cos k \theta_{2}\right)^{2}$, the expected value for log-uniform random variables (19) and the approximation (20) give

$$
\begin{aligned}
\mathbb{E}\left[\exp \left(s_{\boldsymbol{\omega}}\left(\theta_{1}\right)+s_{\boldsymbol{\omega}}\left(\theta_{2}\right)\right)\right] \approx & \left(\prod_{k=2}^{N} \exp \left[\frac{\delta_{k}^{2}}{2}\left(\cos k \theta_{1}+\cos k \theta_{2}\right)^{2}\right]\right) \\
& \times\left(\prod_{k=2}^{N} \exp \left[\frac{\delta_{k}^{2}}{2}\left(\sin k \theta_{1}+\sin k \theta_{2}\right)^{2}\right]\right) \\
= & \prod_{k=2}^{N} \exp \left[\delta_{k}^{2}\left(1+\cos k \theta_{1} \cos k \theta_{2}+\sin k \theta_{1} \sin k \theta_{2}\right)\right] \\
= & \prod_{k=2}^{N} \exp \left[\delta_{k}^{2}\left(1+\cos k\left(\theta_{1}-\theta_{2}\right)\right)\right] \\
= & {\left[\prod_{k=2}^{N} \exp \left(\delta_{k}^{2}\right)\right] \prod_{k=2}^{N} \exp \left[\delta_{k}^{2} \cos k\left(\theta_{1}-\theta_{2}\right)\right] }
\end{aligned}
$$

From (6), and using the normalisation of the variances,

$$
\prod_{k=2}^{N} \exp \left(\delta_{k}^{2}\right)=\exp \left(\delta^{2}\right)=1+\sigma^{2} .
$$

Substituting into (22) and using the covariance of the log-radius (7), we obtain

$$
\mathbb{E}\left[\exp \left(s_{\boldsymbol{\omega}}\left(\theta_{1}\right)+s_{\boldsymbol{\omega}}\left(\theta_{2}\right)\right)\right] \approx\left(1+\sigma^{2}\right) \exp \left[\operatorname{Cov}\left(s_{\boldsymbol{\omega}}\left(\theta_{1}\right), s_{\boldsymbol{\omega}}\left(\theta_{2}\right)\right)\right] .
$$

Finally, substituting into (21) gives

$$
\mathbb{E}\left[r_{\boldsymbol{\omega}}\left(\theta_{1}\right) r_{\boldsymbol{\omega}}\left(\theta_{2}\right)\right] \approx \exp \left[\operatorname{Cov}\left(s_{\boldsymbol{\omega}}\left(\theta_{1}\right), s_{\boldsymbol{\omega}}\left(\theta_{2}\right)\right)\right] .
$$

Now we approximate the second term on the right hand side of (18). Again using (3) we write the radius $\boldsymbol{r}_{\boldsymbol{\omega}}$ in terms of the $\log$-radius $\boldsymbol{s}_{\boldsymbol{\omega}}$,

$$
\mathbb{E}\left[r_{\boldsymbol{\omega}}(\theta)\right]=\frac{1}{\sqrt{1+\sigma^{2}}} \mathbb{E}\left[\exp \left(s_{\boldsymbol{\omega}}(\theta)\right)\right]
$$


Using the Fourier expansion of the log-radius (4),

$$
s_{\boldsymbol{\omega}}(\theta)=\sum_{k=2}^{N} a_{k} \cos k \theta+\sum_{k=2}^{N} b_{k} \sin k \theta
$$

and the independence of the random variables $a_{2}, \ldots, a_{N}$ and $b_{2}, \ldots, b_{N}$, the expected value on the right hand side of (24) decouples into factors:

$$
\mathbb{E}\left[\exp \left(s_{\boldsymbol{\omega}}(\theta)\right)\right]=\left(\prod_{k=2}^{N} \mathbb{E}\left[\exp \left(a_{k} \cos k \theta\right)\right]\right)\left(\prod_{k=2}^{N} \mathbb{E}\left[\exp \left(b_{k} \sin k \theta\right)\right]\right) .
$$

Using the expected value for log-uniform random variables (19) and the approximation (20) in a similar way to above gives

$$
\begin{aligned}
\mathbb{E}\left[\exp \left(s_{\boldsymbol{\omega}}(\theta)\right)\right] & \approx\left[\prod_{k=2}^{N} \exp \left(\frac{\delta_{k}^{2}}{2} \cos ^{2} k \theta\right)\right]\left[\prod_{k=2}^{N} \exp \left(\frac{\delta_{k}^{2}}{2} \sin ^{2} k \theta\right)\right] \\
& =\prod_{k=2}^{N} \exp \left(\frac{\delta_{k}^{2}}{2}\right)=\sqrt{1+\sigma^{2}}
\end{aligned}
$$

where in the last line we again use (23). Finally, substituting into (24) gives

$$
\mathbb{E}\left[\mathrm{r}_{\boldsymbol{\omega}}(\theta)\right] \approx 1 .
$$

The approximation (8) now follows immediately.

\section{Author addresses}

1. M. Ganesh, Department of Applied Mathematics and Statistics, Colorado School of Mines, Golden, CO 80401, USA. mailto:mganeshomines . edu

2. S. C. Hawkins, Department of Mathematics, Macquarie University, Sydney, NSW 2109, Australia.

mailto:stuart. hawkins@mq.edu.au 